\title{
Blackberry polyphenols: review of composition, quantity, and health impacts from in vitro and in vivo studies
}

\author{
Julie A. Robinson ${ }^{\mathrm{a}}$, Julianne E. Bierwirth ${ }^{\mathrm{a}}$, Phillip Greenspan ${ }^{\mathrm{b}}$ and Ronald B. Pegg ${ }^{\mathrm{a}}$ \\ aDepartment of Food Science \& Technology, College of Agricultural and Environmental Sciences, The University of Georgia, 100 Cedar \\ Street, Athens, GA 30602, USA \\ ${ }^{b}$ Department of Pharmaceutical \& Biomedical Sciences, College of Pharmacy, The University of Georgia, 250 W. Green Street, Athens, \\ GA 30602, USA \\ ${ }^{*}$ Corresponding author: Department of Food Science \& Technology, College of Agricultural and Environmental Sciences, The University \\ of Georgia, 100 Cedar Street, Athens, GA 30602, USA. Tel: +1-706-542-1099; Fax: +1-706-542-1050; E-mail: rpegg@uga.edu \\ DOI: $10.31665 / \mathrm{JFB} .2020 .9217$ \\ Received: March 31, 2020; Revised received \& accepted: March 31, 2020 \\ Abbreviations: C3G, cyanidin-3-O-glucoside; DoP, degree of polymerization; DAD, diode array detection; DPPH, 2,2'-diphenyl-1-picryl- \\ hydrazyl; FW, fresh weight; HHDP, hexahydroxydiphenic acid; MI, myocardial infarction; MS, mass spectrometry; NF- $\kappa \mathrm{B}$, nuclear factor \\ kappa-B; PACs, proanthocyanidins; RP-HPLC, reversed-phase high performance liquid chromatography \\ Citation: Robinson, J.A., Bierwirth, J.E., Greenspan, P., and Pegg, R.B. (2020). Blackberry polyphenols: review of composition, quantity, \\ and health impacts from in vitro and in vivo studies. J. Food Bioact. 9: 40-51.
}

\begin{abstract}
Blackberries possess marked concentrations of antioxidant polyphenols, most notably anthocyanins, ellagic acid, ellagitannins, epi/catechin, and proanthocyanidins. While the quantity and type of polyphenols can vary, cyanidin-3-O-glucoside (C3G) is consistently the most abundant polyphenol found in blackberries. Though blackberry antioxidants have not demonstrated significant bioavailability or bioaccessibility in their native form, emerging research suggests that antioxidant metabolites, such as protocatechuic acid derived from C3G, may account for some of the in vivo benefits. Cell studies conducted with blackberry phenolic extracts have demonstrated antiinflammatory, chemopreventive, and neuroprotective effects; whereas, animal models given blackberries have exhibited reduced insulin resistance, attenuated weight gain, and a decrease in inflammatory markers. Blackberry consumption can lead to positive health outcomes. In human health cross-sectional and longitudinal studies, fresh berry consumption has been associated with better long-term insulin resistance, cognitive function, bone density, and cardiovascular function. Research studies, combining in vitro digestion and absorption with targeted cell studies, are being performed to better understand the metabolism and bioactivity of blackberry phenolics. These compounds are not only absorbed by the body, but also can attach to the lining of the digestive tract or be fermented in the colon, contributing to the health outcomes afforded by blackberry consumption.
\end{abstract}

Keywords: Blackberries; Polyphenols; Antioxidants; Anthocyanins; Ellagitannins; Proanthocyanidins.

\section{Introduction}

Blackberries, Rubus spp., are cultivated worldwide and are of growing commercial relevance. Blackberries are usually consumed fresh, but are also made into jams, juices, pies/cobblers, and extracts. Modern consumers have become keenly aware of the potential of functional foods for preventing or slowing chronic disease progression (Olas, 2018). Antioxidant research has demonstrated that increased consumption of polyphenol-rich foods, such as blackberries, can have anticancer, antimutagenic, antimicrobial, anti-inflammatory, and neuroprotective efficacy (Nile and Park, 2014). Blackberries are a source of non-nutritive compounds, such as fiber and polyphenols, as well as nutrients including vitamins, minerals, and sugars (Kaume et al., 2012; Olas, 2018). The 
<smiles>O=C(O)c1ccc(O)c(OC2O[C@H](CO)[C@@H](O)[C@H](O)[C@H]2O)c1</smiles>

a<smiles>O=c1oc2c(O)c(O)cc3c(=O)oc4c(O)c(O)cc1c4c23</smiles><smiles>COc1c(O)ccc(-c2c(O)c(O)c(O)c(O)c2C(=O)O)c1C(=O)O</smiles><smiles>OC[C@H]1OC(Oc2cc3c(O)cc(O)cc3[o+]c2-c2ccc(O)c(O)c2)[C@H](O)[C@@H](O)[C@@H]1O</smiles><smiles>O=c1c(OC2O[C@H](CO)[C@@H](O)[C@H](O)[C@H]2O)c(-c2ccc(O)c(O)c2)oc2cc(O)cc(O)c12</smiles>

Figure 1. Chemical structures of selected non-tannin polyphenolic antioxidants reported in blackberries. (a) protocatechuic acid glucoside; (b) ellagic acid; (c) hexahydroxydiphenic acid (HHDP); (d) cyanidin-3-O-glucoside (C3G); and (e) quercetin-3-O-galactoside.

phenolics most often found in blackberries include anthocyanins, tannins, hydroxybenzoic acids, and flavan-3-ols. Anthocyanins, particularly cyanidin-3-O-glucoside $(\mathrm{C} 3 \mathrm{G})$, are consistently the predominant phenolic antioxidant found in blackberries, and have been shown to have prominent bioactivity (Schulz et al., 2019).

The composition and content of blackberry phenolics can vary due to location, cultivar, and time of harvest. Different varieties grown in the same region have been found to contain different amounts of phenolics (Liao et al., 2020; Fan-Chiang and Wrolstad, 2005). Cultivars harvested later in the year or at greater degrees of ripeness can possess an elevated total phenolics content (TPC) (Liao et al., 2020; Mikulic-Petkovsek et al., 2017; Siriwoharn and Wrolstad, 2004). Not all studies have reported the same finding: Siriwoharn et al. (2004), for instance, did not find an increase in the TPC based on ripeness or harvest time; so, this could be specific to certain growing locations or cultivars. There is a variety of different blackberry categories based on how they grow: these include thorny or thornless as well as erect, semi-erect, or trailing. Depending on which category is investigated, different profiles of the blackberry phenolics will be observed (Kolniak-Ostek et al., 2015). The soil composition also can modify the phenolics content in blackberries. For instance when vermicompost was added to soil, the TPCs and anthocyanin contents were greater (Abud-Archila et al., 2018). Bioactive compounds also increased when the soil was irrigated, and the climate was temperate (Croge et al., 2019).

Current US antioxidant recommendations have focused on studies of vitamins and minerals such as vitamin $\mathrm{C}$, vitamin $\mathrm{E}$, $\beta$-carotene (a precursor to vitamin A), selenium, and zinc (National Center for Complimentary and Integrative Health [NCCIH], 2013). These vitamins and minerals have established dietary intake recom- mendations from the USDA, based on research that demonstrates increased consumption can protect against disease. Antioxidant compounds, including phenolics, do not have established governmental recommendations. Because polyphenols have been shown to ameliorate diseases that arise from reactive oxygen species, there could be a benefit to establishing governmental recommendations for phenolic antioxidants (Olas, 2018). Research on high-dose antioxidant supplementation with vitamin $\mathrm{C}$, vitamin $\mathrm{E}$, and $\beta$-carotene have failed to demonstrate an increase in health benefits, and in some studies have shown harmful effects (National Center for Complimentary and Integrative Health [NCCIH], 2013). Polyphenols can have a pro-oxidant effect under conditions that favor autoxidation, but berry extracts have not been found to have the same potentially toxic health effects, which high doses of other antioxidants have demonstrated (Olas 2018). This review focuses on recent work characterizing blackberry antioxidants as well as the in vitro and in vivo studies conducted to determine their physiological effects.

\section{Phenolic composition of blackberries}

Thousands of phenolic antioxidant compounds exist and are classified into several categories based on structural similarities. For example, Craft et al. (2012) separated phenolics into seven categories: phenolic acids, coumarins, flavonoids, isoflavonoids, stilbenes, lignans, and phenolic polymers (tannins). These phenolic groups differ from each other in functional group placement, or the addition of a chemical moiety as in glycosylation (Craft et al., 2012). Chemical structures of selected non-tannin polyphenolic antioxidants and tannins of blackberries are illustrated in Figures 1 and 2, respectively. 
<smiles>COC(=O)c1cc(O)c(O)c(O)c1-c1c(C(=O)O[C@H]2C(O)O[C@H](COC(=O)c3cc(O)c(O)c(O)c3)[C@H](OC(=O)c3cc(O)c(O)c(O)c3O)[C@H]2OC(=O)c2cc(O)c(O)c(O)c2O)cc(O)c(O)c1O</smiles>

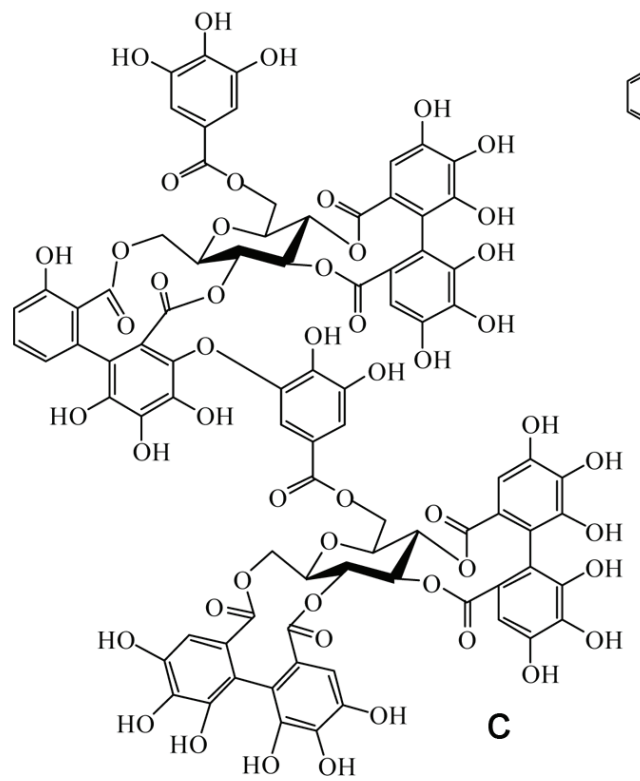<smiles>COC(=O)c1cc(O)c(O)c(O)c1</smiles><smiles>C=C(OC)c1cc(O)c(O)c(O)c1-c1c(C(=O)OC)cc(O)c(O)c1O</smiles>

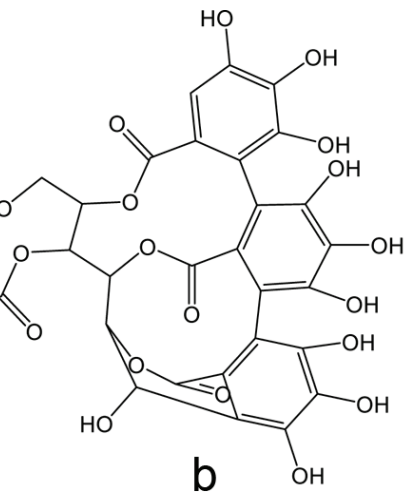

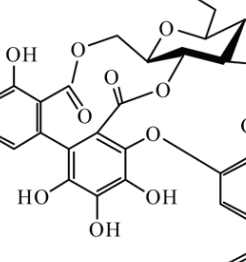<smiles>CC1CCCC(C)C1C</smiles>

b $\mathrm{OH}$<smiles>Oc1cc(O)c2c(c1)O[C@H](c1ccc(O)c(O)c1)[C@H](O)[C@H]2c1c(O)cc(O)c2c1O[C@H](c1ccc(O)c(O)c1)[C@H](O)C2</smiles>

Figure 2. Chemical structures of selected tannin constituents reported in blackberries. (a) pedunculagin; (b) castalagin; (c) sanguiin H-6; (d) lambertianin C; and (e) procyanidin B-type dimer.

The TPC of blackberries varied between 140 and $960 \mathrm{mg}$ gallic acid equivalents (GAE) per $100 \mathrm{~g} \mathrm{FW}$ (Table 1). Gallic acid is only present in small quantities in blackberries, and therefore is not the desired phenolic acid standard for the Folin-Ciocalteu assay; yet, it is the phenolic most often reported for comparison against literature results (Liao et al., 2020; Pantelidis et al., 2007). In terms of blackberries, phenolic acids, flavonoids, and tannins are the most prevalent phenolic classes.

Table 1. Range of total phenolics content, total monomeric anthocyanin content, and total ellagitannins content in blackberries

\begin{tabular}{lllll}
\hline Reference & Location & $\begin{array}{l}\text { TPC (mg GAE/100 } \\
\text { g FW) }\end{array}$ & $\begin{array}{l}\text { TMAC (mg C3G } \\
\text { eq./100 g FW) }\end{array}$ & $\begin{array}{l}\text { Total Ellagitannins } \\
\text { (mg EAE/100 g FW) }\end{array}$ \\
\hline Liao et al., 2020 & Georgia, USA & $409-459$ & $140-150$ & $10.4-27.8$ \\
$\begin{array}{l}\text { Sellappan et al., 2002 } \\
\text { Siriwoharn \& }\end{array}$ & Georgia, USA & $418-555$ & $111-123$ & - \\
Wrolstad, 2004 & Oregon, USA & $822-844$ & $154-225$ & - \\
$\begin{array}{l}\text { Siriwoharn et al., 2004 } \\
\text { Fan-Chiang \& }\end{array}$ & Oregon, USA & & & \\
Wrolstad, 2005 & Oregon, Oklahoma, France, & - & $131-221$ & $20.7-25.4$ \\
Van de Velde et al., 2016 & Macedonia, Mexico and Chile & & $70.3-201$ & - \\
\hline
\end{tabular}

Abbreviations: TPC, total phenolics content; GAE, gallic acid equivalents; FW, fresh weight; TMAC, total monomeric anthocyanins; C3G eq., cyanidin-3-O-glucoside equivalents; $E A E$, ellagic acid equivalents; -, not reported or measured. 


\subsection{Phenolic acids}

The primary phenolic acid in blackberries is ellagic acid; that is, a polyphenol with four resonance stabilized hydroxy groups, classified in the hydroxybenzoic acid family of compounds (Fig. 1). Ellagic acid is commonly glycosylated, and is synthesized during fruit ripening as ellagitannins are degraded (Komorsky-Lovrić and Novak, 2011). Blackberries are a significant source of ellagitannins as well as their hydrolytic breakdown products, ellagic acid and gallic acid. Ellagic acid concentration is highest in the seeds; so, juice processing that homogenizes the seed as well as the flesh and skin retains roughly the same ellagic acid content as that of the whole fruit (Djurić et al., 2014). There is evidence that ellagic acid metabolites can reduce the risk of cancer and mitigate inflammatory response in humans (Espín et al., 2013; Larrosa et al., 2010). Ellagic acid absorbs electromagnetic radiation at wavelengths of 255 and $320 \mathrm{~nm}$, and is often quantified by hydrolyzing the glycoside and then analyzed by reversed-phase high performance liquid chromatography (RP-HPLC) coupled with diode array detection (DAD). Concentrations have been found to vary greatly among blackberry species and cultivars, ranging from 30.0 to $54.8 \mathrm{mg} / 100$ g FW (Djurić et al., 2014; Jakobek and Seruga, 2012; KomorskyLovrić and Novak, 2011; Sellappan et al., 2002). Phenolic acids sometimes present in lesser concentrations may include gallic acid (3.4-6.4 mg/100 g FW), caffeic acid (1.38-3.64 mg/100 g FW), $p$-coumaric acid (0.40-2.08 mg/100 g FW), and ferulic acid (2.99$3.51 \mathrm{mg} / 100$ g FW) (da Rosa et al., 2014; Sellappan et al., 2002).

\subsection{Flavonoids}

\subsubsection{Flavonols and flavan-3-ols}

$(+)$-Catechin and its isomer (-)-epicatechin are consistently the most abundant flavan-3-ols in blackberries. Catechin, like ellagic acid, possesses two aromatic rings with four resonance-stabilized hydroxy groups that can act as hydrogen atom or electron donors. Proanthocyanidins (PACs or condensed tannins) are the polymeric form, which is present in the developing berry and is degraded during ripening to release free $(+)$-catechin and (-)-epicatechin. Catechins are found most abundantly in the skins of the fruit, as opposed to the flesh or seeds (Nile and Park, 2014). There is strong evidence for the positive impact of consuming flavan-3-ols on cognitive and cardiovascular function (Mastroiacovo et al., 2015; Milenkovic et al., 2014). Catechin and epicatechin are detectable by RP-HPLC-DAD at a UV wavelength of $280 \mathrm{~nm}$. Catechin and epicatechin have been reported at concentrations of $378.7 \pm 24.5$ $\mu \mathrm{g} / 100 \mathrm{~g}$ and $448.4 \pm 7.5 \mu \mathrm{g} / 100 \mathrm{~g}$ dry matter, respectively, in blackberries (Schulz et al., 2019).

Flavan-3-ols are one of the phenolic classes, whose concentration is strongly dependent on varietal or growth conditions. As shown in Table 2, catechin was reported in methanolic/ $\mathrm{HCl}$ extracts of Georgia-grown blackberries at concentrations ranging from 266 to $313 \mathrm{mg} / 100 \mathrm{~g} \mathrm{FW}$ (Sellappan et al., 2002), but for berries grown in Ecuador, the catechin levels in an ethyl acetate extract were reported as being undetectable (Jakobek and Seruga, 2012; Vasco et al., 2009). When examining the concentrations of flavan-ols in literature, as well as flavonoids in general, one must be careful to note if an acid hydrolysis step has been employed prior to HPLC analysis. If so, then sugar moieties would be cleaved thereby releasing aglycones and markedly influencing the quantitative findings. In some papers, flavan-3-ol levels are reported as (+)-catechin and $(-)$ epicatechin with no mention of the presence of glycosides or other derivatives, while in other works, they are segregated into free catechin/epicatechin from their derivative forms.

\subsubsection{Anthocyanins}

Anthocyanins contribute to the dark blue color in blackberries, and are the antioxidant class most associated with red berries and fruits. An anthocyanin (i.e., a glycoside in which the sugar is generally attached at the 3-position of the C-ring) forms a $\mathrm{pH}$ dependent 2-phenylbenzopyrylium (flavylium) cation that expresses the deep red color of the blackberries in acidic environments. Anthocyanin intake has been associated with a reduction in insulin resistance, a decreased risk of myocardial infarction (MI), and moderating the inflammatory response (Cassidy et al., 2013; Jennings et al., 2012; 2014).

While the coloration of anthocyanins allows for easy spectrophotometric detection at a wavelength of $520 \mathrm{~nm}$, the diversity of anthocyanins and their derivatives makes isolating individual molecules extremely challenging. Separation is often achieved by RPHPLC and compound identification by mass spectrometry (MS). Fan-Chiang and Wrolstad (2005) determined the anthocyanin contents in eighteen different blackberry varieties from five different locations and found that the total monomeric anthocyanins contents (TMACs) ranged from 70.3 to $201 \mathrm{mg} / 100 \mathrm{~g} \mathrm{FW}$ (Table 1). They reported the presence of five dominant anthocyanins, namely $\mathrm{C} 3 \mathrm{G}$ averaging $82.9 \%$ of the anthocyanins, cyanidin-3-O-rutinoside at $10.2 \%$, cyanidin-3- $O$-xyloside at $2.5 \%$, cyanidin-3- $O$-dioxylglucoside at $2.5 \%$, and cyanidin-3-O-malonylglucoside at $1.9 \%$.

As listed in Table 2, C3G is the chief anthocyanin, comprising as much as $92 \%$ of all anthocyanins in blackberries (Fan-Chiang and Wrolstad, 2005; Niculescu et al., 2013; Ștefănuț et al., 2011; Zhang et al., 2012). Other cyanidin-based anthocyanins have been identified as well, though inconsistently (Table 1). The composition of anthocyanins in blackberries can vary due to location, growing conditions, time of harvest, and differences in cultivars (Fernandes et al., 2014; Liao et al., 2020). Cyanidin is the primary aglycone, comprising as much as $71 \mathrm{mg} / 100 \mathrm{~g} \mathrm{FW}$ in one study (Jakobek and Seruga, 2012). The antiradical activity of the anthocyanins in blackberries, as measured by the DPPH radical assay, is almost seven times that of the combined flavonols and phenolic acids (Jakobek and Seruga, 2012). The results of these bench-top measures imply that most health benefits of blackberries are due to the bioactivity of anthocyanins.

\subsection{Tannins}

Tannins can be classified as either hydrolyzable or PACs. The breakdown of tannins over the course of fruit ripening is responsible for many of the visual and sensory characteristics of mature berries. PACs hydrolyze to form (+)-catechin and (-)-epicatechin. Ellagitannins, an example of a hydrolyzable tannin, can undergo lactonization spontaneously to form hexahydroxydiphenic acid (HHDP, see Fig. 1), a precursor to ellagic acid (Lipińska et al., 2014), which also contributes to tartness. Tannins that remain intact are capable of binding to proteins. This both gives an astringent mouth-feel, as tannins bind to the salivary proteins, and reduces the bioavailability of digested protein (Nile and Park, 2014).

\subsubsection{Proanthocyanidins (PACS)}

PACs, or condensed tannins, are a polymerized form of (+)-catechin and (-)-epicatechin. Their biological significance is largely dependent on their degree of polymerization (DoP); intestinal ab- 
Table 2. Content of dominant phenolic acids, anthocyanins, flavonols, and tannins reported in blackberries in $\mathrm{mg} / 100 \mathrm{~g}$ fresh weight

\begin{tabular}{|c|c|c|c|}
\hline & \multicolumn{3}{|c|}{ Phenolic Acids } \\
\hline & ellagic acid & gallic acid & $p$-coumaric acid \\
\hline Liao et al., 2020 & - & - & 0.55 \\
\hline Fan-Chiang \& Wrolstad, 2005 & - & - & - \\
\hline Kolniak-Ostek et al., 2015 & $0.66-2.71$ & - & $\mathrm{nd}-0.47$ \\
\hline Mertz et al., $2007 *$ & $\mathrm{nd}-0.24$ & $0.06-0.22$ & $0.50-0.76$ \\
\hline Schulz et al., $2019 *$ & - & $0.09-0.18$ & nd -0.03 \\
\hline Sellappan et al., 2002 & $30.01-33.81$ & $4.12-6.42$ & $0.4-2.08$ \\
\hline Siriwoharn et al., 2004 & $1.64-3.62$ & - & - \\
\hline Van de Velde et al., 2016 & - & - & - \\
\hline
\end{tabular}

\begin{tabular}{|c|c|c|c|c|c|c|}
\hline & \multicolumn{6}{|c|}{ Anthocyanins and Flavonols } \\
\hline & $\begin{array}{l}\text { cyanidin-3-O- } \\
\text { glucoside }\end{array}$ & $\begin{array}{l}\text { cyanidin-3-O- } \\
\text { malonylglucoside }\end{array}$ & $\begin{array}{l}\text { cyanidin-3-O- } \\
\text { rutinoside }\end{array}$ & $\begin{array}{l}\text { cyanidin- } \\
\text { 3-O-xyloside }\end{array}$ & $\begin{array}{l}\text { cyanidin-3-O- } \\
\text { dioxalylglucoside }\end{array}$ & $\begin{array}{l}\text { quercetin-3-O- } \\
\text { galactoside }\end{array}$ \\
\hline Liao et al., 2020 & 99.9 & - & - & - & - & 7.06 \\
\hline $\begin{array}{l}\text { Fan-Chiang \& } \\
\text { Wrolstad, } 2005\end{array}$ & $30.65-191.35$ & nd-10.3 & nd-107.3 & nd-22.71 & nd-30 & - \\
\hline Kolniak-Ostek et al., 2015 & $25.15-122.54$ & $0.96-3.34$ & nd-4.66 & nd-6.37 & nd-3.03 & $0.72-2.56$ \\
\hline Mertz et al., $2007 *$ & $45.6-81.6$ & nd-4.8 & nd-75.6 & - & - & - \\
\hline Schulz et al., $2019 *$ & - & - & - & - & - & - \\
\hline Sellappan et al., 2002 & - & - & - & - & - & nd \\
\hline Siriwoharn et al., 2004 & $72.6-81.2$ & $1.12-4.72$ & $2.71-24.2$ & $0.18-6.30$ & $1.91-5.07$ & - \\
\hline Van de Velde et al., 2016 & $91.4-107.3$ & $5.8-7.1$ & - & $0.8-2.9$ & - & $2.94-4.02$ \\
\hline
\end{tabular}

\begin{tabular}{llll}
\hline & & \multicolumn{2}{c}{ Proanthocyanidin Monomer and Tannins } \\
\cline { 2 - 4 } & (-)-epicatechin & lambertianin C & sanguiin H-6 \\
\hline Liao et al., 2020 & - & 5.47 & - \\
Fan-Chiang \& Wrolstad, 2005 & - & - & $1.00-4.82$ \\
Kolniak-Ostek et al., 2015 & - & $2.70-4.56$ & $50.4-294$ \\
Mertz et al., 2007 * & $0.61-0.76$ & $62.4-71.8$ & - \\
Schulz et al., 2019 * & $0.03-0.05$ & - & - \\
Sellappan et al., 2002 & nd & - & - \\
Siriwoharn \& Wrolstad, 2004 & $1.43-4.41$ & - & - \\
Van de Velde et al., 2016 & - & $3.8-4.7$ & - \\
\hline
\end{tabular}

Abbreviations: -, not reported or measured; nd, not detectable but looked for; ${ }^{*}$, results reported on a dry weight have been converted to fresh weight according to the mass balance details.

sorption of PAC dimers is less than $10 \%$, and those with a DoP greater than 4 units are not absorbed at all (Ou and $\mathrm{Gu}, 2014)$. Unfortunately, PACs appear not to hydrolyze or degrade significantly to form monomers during human digestion (Rios et al., 2002). Ou and $\mathrm{Gu}(2014)$ reported that PACs are digested by intestinal microbes to form a series of bioavailable aromatic acid metabolites. While this may be the primary health-promoting mechanism, this digestion also decreases with increasing DoP. Despite absorption limitations, studies have posited that PACs may be beneficial to health by protecting the alimentary canal from oxidative stress and carcinogenesis (Manach et al., 2005). Amongst six species of Mexican blackberries, PAC oligomers were found ranging from 2 to 6 catechin/epicatechin subunits, with measurement of a PAC content averaging 3.32 to $5.37 \mathrm{~g}$ catechin equivalents/100 g FW. (Cuevas-Rodríguez et al., 2010).

\subsubsection{Hydrolyzable tannins}

An ellagitannin (a class of hydrolyzable tannins) is defined by the 
formation of an ester bond between HHDP and a monosaccharide. Ellagitannins exist frequently in nature as high-molecular-weight polymers, but they are highly susceptible to changes including polymerization to form higher DoP oligomers or hydrolysis by intestinal microorganisms to form beneficial urolithins (TomásBarberán et al., 2014). The study of Tomás-Barberán et al. (2014) found inter-subject variability in the metabolites formed from ellagic acid and ellagitannins, suggesting that the microbial diversity of the intestines influences their bioactivity. Like ellagic acid, ellagitannins exhibit anti-inflammatory and anti-carcinogenic properties (Espín et al., 2013; Larrosa et al., 2010). Blackberry ellagitannins are primarily a mixture of sanguiin $\mathrm{H}-6 /$ lambertianin $\mathrm{A}$ and lambertianin $\mathrm{C}$ with total ellagitannin contents ranging from 23 to $343 \mathrm{mg} / 100 \mathrm{~g} \mathrm{FW}$, making them relatively abundant when compared to other fruits (Hager et al, 2008; 2010). Like ellagic acid, Hager et al. (2010) found the majority of ellagitannins in the seeds and that traditional juicing can reduce the ellagitannin content by 70 to $82 \%$.

\section{Bioavailability and bioaccessibility of blackberry phenolics}

Crucial to the understanding of any functional food or phytochemical is the digestion and absorption process, which the nutrients and bioactives undergo. Research often focuses on biomolecules of food that are absorbed from the digestive tract to the bloodstream; nonetheless, constituents that are not absorbed should not be discounted in terms of providing a physiological function. Examining and perceiving how polyphenols, especially anthocyanins, breakdown during digestion and interact with intestinal lining and gut microbiota might possibly explain the health benefits afforded by consuming blackberries, even though the measured bioavailability is low (Fernandes et al., 2014). Though they may not interact with other systemic organ systems, these constituents may still provide a benefit to the epithelial lining of the alimentary canal (Manach et al., 2005). Most research investigating the effect of foods and food components on cultured cells does not factor in the effect of in vitro digestion and absorption. Such studies determine the potential health effects of phenolics that may never reach the intestinal epithelium or may not be absorbed into the bloodstream. Further, they habitually dismiss the role of the metabolism of ingested phenolic compounds (Bowen-Forbes et al., 2010; Calvo-Castro et al., 2013). Other studies ignore matrix effects by assessing the absorption of purified compounds, often at higher-than-relevant concentrations (Serra et al., 2013). These can address the cellular response to a particular phenolic compound, but they should be framed within an understanding of the physiological concentration at which that chemical would be exposed to similar cells during digestion in humans. Nonetheless, an understanding of the chemical modifications that take place during digestion as well as intestinal absorption kinetics is essential. This review will cover the research on the digestion and absorption of blackberries, as well as its isolated phenolic constituents.

\subsection{In vitro digestion and absorption}

\subsubsection{In vitro digestion methodology}

Human digestion varies from person to person; thus, there is not yet one accepted standard for the methodology of in vitro digestion studies (Carbonell-Capella et al., 2014). The majority of in vitro digestion trials include the following steps: grinding or homogenization, oral phase, gastric phase, small intestine phase and isolation of the digesta as well as its uptake by cells. The effect of colonic metabolism will be discussed in section 3.2.2. As explained by Carbonell-Capella (2014), methodologies in studies can differ at every step in the process, including level of sample homogenization, incubation time for each phase, and isolation method for the resulting digesta. Varying the enzyme mixture and concentration can have a profound impact on the results obtained. While gastric pepsin has seemingly no effect on the phenolic composition, $\alpha$-amylase may conjugate with anthocyanins and reduce their bioavailability (Wiese et al., 2009). The condition of the sample prior to digestion influences the result as well. For instance, a homogenized sample may show increased bioavailability, but this does not accurately mimic human mastication patterns like for a study in which the sample is ground (Alminger et al., 2014). A more recent digestion study followed in vitro digestion with gut microbiota fermentation to mimic the environment in which polyphenols and their derivatives undergo in the colon. The results showed improved antidiabetic and antioxidant activities compared to in vitro gastric and duodenal digestion alone (Gowd et al., 2018).

A relatively simple and inexpensive way to mimic intestinal absorption is via dialysis tubing, in which a selectively permeable membrane separates the theoretically absorbed molecules from unabsorbed colon-bound ones (Tavares et al., 2012; 2013). More complex systems use Caco-2 cells, a line of human colon cancer cells differentiated to express intestinal epithelial properties. While a Caco-2 system may be more representative of human absorption, laboratory conditions, cost, amount of time required, and the necessary equipment can make it prohibitive (Carbonell-Capella et al., 2014).

\subsubsection{In vitro digestion and absorption of blackberries and relevant compounds}

An in vitro digestion study (Tavares et al., 2013), in which blackberries from Portugal underwent digestion and simulated absorption at physiologically relevant concentrations, reported a 83 to $95 \%$ post-absorption reduction in antioxidant capacity with a 94.5 to $99.5 \%$ reduction in the TPC. In another study by the same authors, the pre-absorption reduction in TPC and antioxidant capacity was found to be 93 and $49.4 \%$, respectively (Tavares et al., 2012). The reduced antioxidant capacity of dietary phenolics before absorption can result from oxidation, non-covalent binding with intestinal proteins, and transformation into glucuronidated, sulfated, or methylated metabolites. Noteworthy is that anthocyanins have been found to be moderately resistant to such modifications (Carbonell-Capella et al., 2014; Yang et al., 2011). Tavares et al. (2013) also noted that wild blackberry cultivars best retained their antioxidant capacity after digestion as compared to commercial cultivars.

Tomas et al. (2020) examined how the phenolic profile of blackberry puree changed depending on the fiber content added using an in vitro digestion and fermentation study. These researchers found that the interaction with the fiber during digestion lowered the TPC and changed the composition of the free- and bound-phenolics in the sample similarly to the study of Tavares et al. (2013). Furthermore, they found that a potential benefit of the added fiber was that anthocyanins were not bioavailable during gastric and duodenal digestion, but were found to offer an increased antioxidant potential in the large intestine (Tomas et al., 2020).

In vitro digestion studies are difficult to perform because polyphenols will be absorbed differently depending on the food matrix 
in question, and can bind to the lining of the gastrointestinal tract. Surveys on the bioavailability of individual polyphenolic classes find that most abundant dietary components are not necessarily the best absorbed. As discussed earlier, molecular weight/size often plays a critical role in absorption. In general, small molecules such as phenolic acids and catechins are all easily absorbed by the epithelium, whereas large and highly polymerized PACs and ellagitannins are not (Carbonell-Capella et al., 2014).

C3G and other anthocyanins are often the molecules of interest in studies on the health effects of berry phenolics. Research findings have repeatedly shown poor absorption kinetics for $\mathrm{C} 3 \mathrm{G}$ and associated anthocyanins, but yet increased intake is still associated with improved health outcomes (Cassidy et al., 2013; Jennings et al., 2014). Degradation products that occur in the small intestine, particularly the formation of protocatechuic acid, phloroglucinaldehyde and metabolites thereof, appear to be the absorbed bioactives of interest (Carbonell-Capella et al., 2014; Kay et al., 2009)

\subsection{In vivo digestion and absorption}

\subsubsection{In vivo digestion of blackberries}

In a simple bioavailability study, eight healthy subjects consumed $180 \mathrm{~g}$ of blackberries and were subsequently analyzed for plasma and urine anthocyanin concentrations. $\mathrm{C} 3 \mathrm{G}$ was metabolized primarily to peonidin- $O$-glucuronide. The concentrations of $\mathrm{C} 3 \mathrm{G}$ and its metabolites in the plasma ( 5 to $20 \mathrm{nmol} / \mathrm{L}$ ) and the urine $(0.08 \%$ of ingested mass) were low compared to the amount consumed (AlGamdi, 2013). A subsequent study was performed on five ileostomy subjects to determine the influence of digestion and absorption via the small intestine: unaltered blackberry anthocyanins were recovered at $5.1 \%$ of the initial dose (AlGamdi, 2013). In an experiment to determine the distribution of anthocyanins and their derivatives in target organs, sixteen rats were fed a blackberry-enriched diet for twelve days. Urine, plasma, bladder, prostate, heart, testes, and adipose tissue were analyzed for $\mathrm{C} 3 \mathrm{G}$ and total anthocyanins concentration. The highest levels of $\mathrm{C} 3 \mathrm{G}$ and anthocyanins were found in the bladder $(1.37 \mathrm{nmol} \mathrm{C} 3 \mathrm{G} / \mathrm{g}$ tissue and 2.37 nmol C3G equivalents/g tissue, respectively). Very low concentrations were recovered from blood serum and urine. The second highest concentration of anthocyanins was found in the prostate (Felgines et al., 2009). Because studies have shown health benefits from blackberry consumption, these rather low concentrations of phenolics have either significant bioactivity or there are other ways in which blackberry components impart health benefits.

\subsubsection{In vivo digestion of isolated phenolics of interest}

The following paragraphs summarize research on the bioavailability and bioabsorbability of isolated phenolic molecules commonly found in blackberries, which include epi/catechin, ellagic acid, ellagitannins, PACs, and anthocyanins. Epi/catechins and other flavan-3-ols are partially absorbed in the small intestines, but the majority travel to the large intestine where they are broken down into absorbable phenolic acids by colonic bacteria (Calani et al., 2012). Free ellagic acid can be absorbed beginning in the gastric compartment and continuing in the small intestine. Ellagitannins, on the other hand, must first be hydrolyzed in the small intestine to form ellagic acid and then transformed by intestinal microbiota to dibenzopyranone metabolites - urolithin A and B - which are absorbed in the colon and undergo glucuronidation (Lipińska et al.,
2014). Similarly, PACs with high DoP were not shown to degrade significantly during gastrointestinal digestion in rats, but were metabolized by colonic bacteria to form phenolic acids and other metabolites; this may account for their reported bioactivity (Ou and $\mathrm{Gu}, 2014)$. These authors reported absorption rates of $10 \%$ and less for PAC dimers, trimers, and tetramers with decreasing absorption as the DoP increases. To further emphasize the role of microbiota-associated digestion, a study comparing C $3 \mathrm{G}$ metabolism in rats with translocated human intestinal bacteria versus their 'clean' equivalents found over twice the recovery of $\mathrm{C} 3 \mathrm{G}$ and its byproducts in the urine of the bacteria-rich group (Hanske et al., 2013). Digestion and absorption research increasingly points to intestinal microorganisms and their key role in metabolizing unabsorbed polyphenols into biologically active substrates.

$\mathrm{A}^{13} \mathrm{C}$ tracer can be used to help elucidate the metabolic pathway of a specific bioactive. Five hundred milligrams of ${ }^{13} \mathrm{C}$-labeled $\mathrm{C} 3 \mathrm{G}$ (with three ${ }^{13} \mathrm{C}$ atoms on the A-ring and two ${ }^{13} \mathrm{C}$ atoms on the B-ring) were fed to eight male participants with urine, blood, breath, and feces collected over $48 \mathrm{~h}$ (Czank et al., 2013). Urinary and blood concentrations peaked within an hour of consumption. Maximum elimination occurred from the breath at $6 \mathrm{~h}$ and in feces between 6 and $24 \mathrm{~h}$. At its height, fecal elimination was more than four times that of urinary elimination. Altogether, twenty-four metabolites of C3G were identified by HPLC-MS. The primary metabolites at each stage were phenylacetic and phenylpropenoic acids. Other metabolite classes included phase II conjugates possessing methylated and glucuronidated moieties, degradants such as protocatechuic acid and phloroglucinaldehyde, conjugates of protocatechuic acid including vanillic acid and 2-hydroxy-4-methoxybenzoic acid, and hippuric acid. The most prevalent $\mathrm{C} 3 \mathrm{G}$ metabolites in serum were phase II conjugates of protocatechuic acid. The minimum bioavailability of C3G was $12.38 \%$ in this study (Czank et al., 2013). These results further confirm the degradation and metabolism of $\mathrm{C} 3 \mathrm{G}$, accounting for the low recovery of the parent compound in urine and its low concentrations in serum.

While only a small percentage of polyphenols are absorbed into the blood stream, polyphenols and their metabolites could also potentially attach to the intestinal epithelium and be important for the health of beneficial bacteria in the colon. The effect of polyphenols on the gut microbiome has just recently begun to be investigated in detail. For example, Ma and Chen (2020) determined that polyphenolic supplementation changed the types of bacteria found in the large intestine without significantly increasing or decreasing total bacterial counts. Beneficial bacteria such as Lactobacillus spp. and Bifidobacterium spp. increased with polyphenolic supplementation by 220 and $56 \%$, respectively. Other bacterial species such as Eubacterium, Bacteroides, Prevotella, Enterococcus, and Enterobacteria were not consistently changed with polyphenolic supplementation (Ma and Chen, 2020).

\section{In Vitro cell studies}

Berry extracts have been employed in cultured in vitro cell studies in an effort to understand their potential for preventing or slowing the progression of cancer, diabetes, neurodegeneration, inflammation, and oxidative damage. It is important to note that, unless otherwise stated, the extracts investigated were undigested and unabsorbed, and therefore represent an incomplete estimation of the influence of orally consumed blackberries on target cells. Furthermore, different types of cell lines have been used in assays and include Caco-2, HepG2, and BV-2 microglia cells, depending on 
whether the study was focused on absorption or neuroprotective properties (Kellett et al., 2018; Ma et al., 2018).

\subsection{Chemoprevention}

Research investigations on the effects of blackberry extracts on cancer progression are numerous. In a study of Jamaican- and Michigan-grown blackberry as well as raspberry fruits, Jamaican blackberry extracts at a concentration of $250 \mu \mathrm{g} / \mathrm{mL}$ were found to significantly reduce the proliferation of breast, lung, colon and gastric cancer cells by $24,54,50$, and $57 \%$, respectively (BowenForbes et al., 2010). Promising findings have been documented for leukemia; that is, over $24 \%$ of cells exposed to blackberry extracts at $100 \mu \mathrm{g} / \mathrm{mL}$ were converted to the $\mathrm{G} 2 / \mathrm{M}$ phase of the cell cycle (Zunino et al., 2010). Similarly, cells incubated with a Costa Rica blackberry juice extract initiated apoptosis of UVB-damaged keratinocytes to a greater extent than that of control cells (CalvoCastro et al., 2013). Suppression of the inflammatory macrophage cytokine tumor necrosis factor-alpha, which can exacerbate cell proliferation and metastasis in cancers with transcriptional dysregulation by nuclear factor kappa-B (NF- $\mathrm{kB})$, and AP-1 transcription factors, is a postulated mechanism for these effects (Ding et al., 2006; Milenkovic et al., 2014).

\subsection{Inflammation}

Fermented blueberry- and blackberry-mixed juices at $100 \mu \mathrm{M} \mathrm{C} 3 \mathrm{G}$ equivalents concentration were able to drastically decrease inflammation by as much as $80 \%$ in murine RAW 264.7 macrophage cells, as measured by cyclooxygenase- 2 and NF- $\kappa B$ inhibition (Johnson et al., 2013). C3G has shown even greater anti-inflammatory activity over some anti-inflammatory drugs. Noteworthy is that $\mathrm{C} 3 \mathrm{G}$ reduced cytokine-induced inflammation in human intestinal HT-29 cells at a lower concentration than that of 5-aminosalicylic acid (Serra et al., 2013). Ellagitannins from blackberries have been reported to reduce inflammation via inhibition of the NF- $\mathrm{KB}$ pathway in gastric cells (Sangiovanni et al., 2013).

\subsection{Neurodegeneration}

The influence of digestion on the in vitro cellular response to blackberry extracts has been studied when neuroblastoma cells were exposed to $\mathrm{H}_{2} \mathrm{O}_{2}$ to mimic age-related neurodegeneration and then treated with either raw or digested, as well as dialyzed, blackberry extracts at physiological concentrations. The results showed not only a protective effect at the lowest dose of the digested extract $(1.5 \mu \mathrm{M})$, but also no protective effect from the undigested fraction, even at the highest concentration employed (Tavares et al., 2012). The enhanced response of cells to the digested fraction suggests an adaptive response by mammalian cells to favor biologically available polyphenols. This finding matches later research by the same investigators, who found an enhanced effect for digested wild blackberry species in preventing neuroblastoma cells from oxidative neurodegeneration (Tavares et al., 2013).

\subsection{Diabetes and obesity}

When carbohydrates are ingested they are broken down by enzymes, including glucosidases and amylases, into monosaccharides. A type-2 diabetic could possibly prevent hyperglycemic complications by having the ingested carbohydrates remain intact, which Spínola et al. (2019) was able to demonstrate in vitro. When exposed to blackberry extracts (especially those rich in $\mathrm{C} 3 \mathrm{G}$ ), glucosidases, but not amylases, were significantly inhibited. Protection from oxidative stress was also improved by a blackberry extract (Spínola et al., 2019).

\subsection{Considerations}

The duration of sample exposure presents a challenge to all cell studies. For example, cancer cells are exposed to high concentrations of anthocyanins for upwards of $24 \mathrm{~h}$ after one hour of consumption (Bowen-Forbes et al., 2010; Czank et al., 2013). This would imply that chronic and sustained consumption of berries, or at least consumption concurrent with events like initiation of inflammatory response, is necessary in order to achieve the desired benefit. Of further note, while in vitro cell studies test the sample extracts for cytotoxicity, the lack of deleterious effects from phenolics does not imply that physiological dosage would be the same. One study cites $4 \mu \mathrm{M}$ as being the highest physiological dose of serum polyphenols; yet, many studies have used as much as $100 \mu \mathrm{M}$ (Manach et al., 2005; Tavares et al., 2012). The employment of higher than normal concentrations with longer than typical exposure times may mimic repeated long-term consumption effects, or it may simply create enough statistical power to achieve significant findings in, say, a model cell experimental system. Ideally, future cell studies will take into account the in vitro digestive process and the bioactivity of the absorbed metabolites.

\section{Animal studies}

Next to human clinical trials, animal studies are the best way in which to explore the bioactivity of blackberry extracts. Blackberry juices and extracts administered to mice and rats have had many effects including reductions in insulin resistance, inflammation, oxidative stress, and weight gain. In an assessment of the antihyperglycemic effect of berries, blackberry phenolic extracts were administered to diabetic rats over the course of five weeks; the average serum glucose concentrations decreased significantly from 360 to $270 \mathrm{mg} / \mathrm{dL}$ (Ștefănuț et al., 2013).

In a study on gastric inflammation, rats treated orally for ten days with blackberry ellagitannins showed reduced peptic ulcers, protection from ethanol-induced stress, and inhibition of the NF- $\kappa$ B pathway (Sangiovanni et al., 2013). A comparison of the antioxidant potential of ellagitannins, anthocyanins, and whole blackberry aqueous extracts was performed by feeding healthy rats for 35 days. Antioxidation markers such as catalase, glutathione, and superoxide dismutase levels were determined in the serum, erythrocytes, liver, kidney, spleen, and brain. The findings indicated an antioxidant response to blackberry consumption (Hassimotto and Lajolo, 2011): this result is supported by similar research in oxidatively stressed mice (Cho et al., 2011; Hassan and Abdel-Aziz, 2010). Other effects of blackberry consumption on rats and mice include a shift in the microflora composition of mice fed blackberry powder (Park et al., 2013) and attenuated weight gain as well as inflammation in post-ovariectomized female rats consuming whole blackberries (Kaume et al., 2012). When rats were given a blackberry extract before being induced into a manic state with ketamine, brain interleukin proteins, markers of the immune response, were reduced (Chaves 
et al., 2020). These studies indicate that at physiological doses, blackberries and their extracts can have a multitude of positive influences on a living organism.

\section{Human studies}

There exist human studies on blackberry consumption; however, most are not clinical trials, rather they are cross-sectional and longitudinal studies of dietary patterns along with a number of habits and risk factors. This section describes research specific to blackberries, but also research on overall berry consumption, anthocyanin intake, and cyanidin supplementation.

\subsection{Anti-hypoglycemic effects}

Diabetes complications can be exacerbated by oxidative stress. Antioxidant consumption has been reported to help prevent such complications from worsening (Solverson et al., 2018). These authors tested seventeen overweight or obese men by feeding them either $600 \mathrm{~g}$ of blackberries, or carbohydrate-matched gelatin of the same caloric content. The results showed that fat oxidation and insulin sensitivity increased for the men fed blackberries, with younger subjects demonstrating the best improvement (Solverson et al., 2018). A cross sectional study of 1,997 women investigated flavonoid consumption broken down by class against diabetesrelated markers such as fasting glucose, insulin, and C-reactive protein. Of all classes of flavonoids, only anthocyanins and flavones imparted beneficial effects; that is, increased anthocyanin consumption correlated with reduced peripheral insulin resistance, reduced C-reactive protein, reduced insulin levels, and reduced inflammation (Jennings et al., 2014). A 2012 analysis of participants in the Nurses Health Studies (NHS and NHS II) combined with the Health Professionals Follow-Up Study confirmed this correlation. With a cohort of $>200,000$ healthy individuals, 12,611 cases of type- 2 diabetes were documented over the duration of the respective studies. Of all of the flavonoid classes and subclasses, only anthocyanins were associated with a reduced risk in developing type-2 diabetes (Wedick et al., 2012).

\subsection{Cognitive function}

Research on the relationship between blackberry consumption and cognitive function in humans is extremely limited. In a study of the long-term cognitive function of $>16,000$ aged $70^{+}$NHS participants, dietary analysis identified that greater berry intake was associated with slower loss of cognition due to age. Participants who consumed large amounts of berries delayed that cognition loss by roughly 2.5 years over the course of the 6-year study (Devore et al., 2012).

\subsection{Bone health}

Bone loss occurs naturally over time, but can be exacerbated by lifestyle choices. In a clinical trial of female post-menopausal smokers, the women were assigned to different levels and sources of berry consumption. Participants were analyzed at $0,3,6$, and 9 months for oxidative stress, bone density measurements, and inflammatory markers. Of the berries consumed in the study, blackberries were the only type that exhibited low-level prevention of smoking-induced bone loss (Kaume et al., 2014). In a twin-study encompassing 3,160 participants, total flavonoid and sub-categorical consumption acquired from food-frequency questionnaires was analyzed along with bone density at the spine and hip. Total flavonoid intake was deemed to be positively associated with higher bone mineral density. The strongest association among the sub-categories of flavonoids was with anthocyanins, which demonstrated a 3.4 and $3.1 \%$ greater mineral density at the spine and hip, respectively. The second greatest association was found among flavones, while all other subcategories had non-significant results (Welch et al., 2012).

\subsection{Heart health}

The Nurses' Health Study population was used once again, this time to demonstrate the correlation between flavonoid intake and risk of MI. Out of 93,600 healthy participants aged 25 to 42 years, 405 cases of MI were documented over the 18-year follow up. Correlation with food frequency questionnaires found that increased intake of anthocyanin-rich foodstuffs significantly reduced the risk of MI, with high anthocyanin consumers approximately $32 \%$ less likely to suffer MI (Cassidy et al., 2013). Another study found that measures of arterial stiffness and blood pressure were improved among high anthocyanin and flavone consumers in a cross sectional study of 1,898 twins (Jennings et al., 2012).

\section{Conclusions}

Blackberries possess a rich array of potentially bioactive constituents outside those with current dietary recommendations. C3G is the most studied and most understood of these phytochemicals, and may exhibit a greater influence on the health of the consumer than any other individual molecule found in the fruit. Despite the low reported absorption of many key bioactives in their native form, modifications that occur during digestion may, in fact, improve intestinal absorption and effectiveness. Studies that do not consider digestion and absorption of phenolic constituents may actually see a diminished effect in the raw, undigested samples as compared to the breakdown products and metabolites.

In vitro cell studies, as well as animal studies, have shown significant benefits of blackberry phenolics, including apoptosis of carcinogenic cells, reduced inflammation, lowered blood sugar, and decreased weight gain. Anthocyanins, as a class, are consistently the most efficacious of blackberry phenolics in human crosssectional studies, longitudinal studies, and controlled clinical trials. Human studies have correlated anthocyanin consumption also with improved bone density, improved cardiovascular functioning, improved memory, and reduced insulin resistance. Further research to understand the influence of blackberry phenolics on cultured cell systems should attempt to mimic the modifications that occur in the alimentary canal employing relevant physiological concentrations.

Understanding of the function of blackberries in human health would be greatly augmented by additional randomized controlled clinical trials. Ideally such studies would supplement the diet with isolated blackberry phenolics, or else control for calories and other nutrients found in blackberries, in order to determine the influence of the phenolic antioxidants alone. Despite gaps in current research, the existing data demonstrates the potential for blackberries as a healthy dietary constituent with multi-functionality in its influence on chronic disease progression (Van de Velde et al., 2016). 


\section{References}

Abud-Archila, M., Luján-Hidalgo, M.C., López-Pérez, J.M., Ordaz-Rivera, J., Ruíz-Valdiviezo, V.M., Oliva-Llaven, M.Á., and Gutiérrez-Miceli, F.A. (2018). Growth and fruit chemical characteristics of blackberry ( $R u$ bus fruticosus) cultivated with vermicompost, Glomus mosseae, and phosphate rock. Compost Sci. Util. 26: 225-231.

AlGamdi, N.A. Characterisation of polyphenolic compounds in herbal tea and berries: in vivo and in vitro studies on the bioavailability of anthocyanins. PhD dissertation. University of Glasgow. Retrieved from http://theses.gla.ac.uk/4768/.

Alminger, M., Aura, A.-M., Bohn, T., Dufour, C., El, S.N., Gomes, A., Karakaya, S., Martínez-Cuesta, M.C., McDougall, G.J., Requena, T., and Santos, C.N. (2014). In vitro models for studying secondary plant metabolite digestion and bioaccessibility. Compr. Rev. Food Sci. Food Saf. 13: 413-436.

Bowen-Forbes, C.S., Zhang, Y., and Nair, M.G. (2010). Anthocyanin content, antioxidant, anti-inflammatory and anticancer properties of blackberry and raspberry fruits. J. Food Compos. Anal. 23: 554-560.

Calani, L., Del Rio, D., Callegari, M.L., Morelli, L., and Brighenti, F. (2012). Updated bioavailability and $48 \mathrm{~h}$ excretion profile of flavan-3-ols from green tea in humans. Int. J. Food Sci. Nutr. 63: 513-521.

Calvo-Castro, L., Syed, D.N., Chamcheu, J.C., Vilela, F.M.P., Pérez, A.M., Vaillant, F., Rojas, M., and Mukhtar, H. (2013). Protective effect of tropical highland blackberry juice (Rubus adenotrichos Schltdl.) against UVB-mediated damage in human epidermal keratinocytes and in a reconstituted skin equivalent model. Photochem. Photobiol. 89: 1199-1207.

Carbonell-Capella, J.M., Buniowska, M., Barba, F.J., Esteve, M.J., and Frígola, A. (2014). Analytical methods for determining bioavailability and bioaccessibility of bioactive compounds from fruits and vegetables: A review. Compr. Rev. Food Sci. Food Saf. 13: 155-171.

Cassidy, A., Mukamal, K.J., Liu, L., Franz, M., Eliassen, A.H., and Rimm, E.B. (2013). High anthocyanin intake is associated with a reduced risk of myocardial infarction in young and middle-aged women. Circulation 127: 188-196.

Chaves, V.C., Soares, M.S.P., Spohr, L., Teixeira, F., Vieira, A., Constantino, L.S., Dal Pizzol, F., Lencina, C.L., Spanevello, R.M., Freitas, M.P., Simões, C.M.O., Reginatto, F.H., and Stefanello, F.M. (2020). Blackberry extract improves behavioral and neurochemical dysfunctions in a ketamine-induced rat model of mania. Neurosci. Lett. 714: 134566.

Cho, B.O., Ryu, H.W., Jin, C.H., Choi, D.S., Kang, S.Y., Kim, D.S., Byun, M.-W., and Jeong, I.Y. (2011). Blackberry extract attenuates oxidative stress through up-regulation of Nrf2-dependent antioxidant enzymes in carbon tetrachloride-treated rats. J. Agric. Food Chem. 59: 1144211448

Craft, B.D., Kerrihard, A.L., Amarowicz, R., and Pegg, R.B. (2012). Phenolbased antioxidants and the in vitro methods used for their assessment. Compr. Rev. Food Sci. Food Saf. 11: 148-173.

Croge, C.P., Cuquel, F.L., Pintro, P.T.M., Biasi, L.A., and De Bona, C.M. (2019). Antioxidant capacity and polyphenolic compounds of blackberries produced in different climates. HortScience 54: 2209-2213.

Cuevas-Rodríguez, E.O., Yousef, G.G., García-Saucedo, P.A., López-Medina, J., Paredes-López, O., and Lila, M.A. (2010). Characterization of anthocyanins and proanthocyanidins in wild and domesticated Mexican blackberries (Rubus spp.). J. Agric. Food Chem. 58: 7458-7464.

Czank, C., Cassidy, A., Zhang, Q., Morrison, D.J., Preston, T., Kroon, P.A., Botting, N.P., and Kay, C.D. (2013). Human metabolism and elimination of the anthocyanin, cyanidin-3-glucoside: ${ }^{13} \mathrm{C}$-tracer study. Am. J. Clin. Nutr. 97: 995-1003.

da Rosa, C.G., Borges, C.D., Zambiazi, R.C., Rutz, J.K., da Luz, S.R., Krumreich, F.D., Benvenutti, E.V., and Nunes, M.R. (2014). Encapsulation of the phenolic compounds of the blackberry (Rubus fruticosus). LWT - Food Sci. Technol. 58: 527-533.

Devore, E.E., Kang, J.H., Breteler, M.M.B., and Grodstein, F. (2012). Dietary intakes of berries and flavonoids in relation to cognitive decline. Ann. Neurol. 72: 135-143.

Ding, M., Feng, R., Wang, S.Y., Bowman, L., Lu, Y., Qian, Y., Castranova, V., Jiang, B-H., and Shi, X. (2006). Cyanidin-3-glucoside, a natural product derived from blackberry, exhibits chemopreventive and chemothera- peutic activity. J. Biol. Chem. 281: 17359-17368.

Djurić, M., Maškovic, P., Murtić, S., Veljković, B., Ćurčić, S., Paunović, G. and Rakočević, L.B. (2014). Quantitation of ellagic acid in blackberries. Hem. Ind. 68: 241-245.

Espín, J.C., Larrosa, M., García-Conesa, M.T., and Tomás-Barberán, F. (2013). Biological significance of urolithins, the gut microbial ellagic acid-derived metabolites: The evidence so far. J. Evidence-Based Complementary Altern. Med. 2013: 270418.

Fan-Chiang, H-J., and Wrolstad, R.E. (2005). Anthocyanin pigment composition of blackberries. J. Food Sci. 70: C198-C202.

Felgines, C., Texier, O., Garcin, P., Besson, C., Lamaison, J.-L., and Scalbert, A. (2009). Tissue distribution of anthocyanins in rats fed a blackberry anthocyanin-enriched diet. Mol. Nutr. Food Res. 53: 1098-1103.

Fernandes, I., Faria, A., Calhau, C., de Freitas, V., and Mateus, N. (2014). Bioavailability of anthocyanins and derivatives. J. Funct. Foods 7: $54-66$.

Gowd, V., Bao, T., Wang, L., Huang, Y., Chen, S., Zheng, X., Cui, S., and Chen, W. (2018). Antioxidant and antidiabetic activity of blackberry afte gastrointestinal digestion and human gut microbiota fermentation. Food Chem. 269: 618-627.

Hager, T.J., Howard, L.R., and Prior, R.L. (2010). Processing and storage effects on the ellagitannin composition of processed blackberry products. J. Agric. Food Chem. 58: 11749-11754.

Hager, T.J., Howard, L.R., Liyanage, R., Lay, J.O., and Prior, R.L. (2008). Ellagitannin composition of blackberry as determined by HPLC-ESI-MS and MALDI-TOF-MS. J. Agric. Food Chem. 56: 661-669.

Hanske, L., Engst, W., Loh, G., Sczesny, S., Blaut, M., and Braune, A. (2013). Contribution of gut bacteria to the metabolism of cyanidin 3-glucoside in human microbiota-associated rats. Br. J. Nutr. 109: 14331441

Hassan, H.A., and Abdel-Aziz, A.F. (2010). Evaluation of free radical-scavenging and anti-oxidant properties of black berry against fluoride toxicity in rats. Food Chem. Toxicol. 48: 1999-2004.

Hassimotto, N.M.A., and Lajolo, F.M. (2011). Antioxidant status in rats after long-term intake of anthocyanins and ellagitannins from blackberries. J. Sci. Food Agric. 91: 523-531.

Jakobek, L., and Seruga, M. (2012). Influence of anthocyanins, flavonols and phenolic acids on the antiradical activity of berries and small fruits. Int. J. Food Prop. 15: 122-133.

Jennings, A., Welch, A.A., Fairweather-Tait, S.J., Kay, C., Minihane, A.-M., Chowienczyk, P., Jiang, B., Cecelja, M., Spector, T., Macgregor, A., and Cassidy, A. (2012). Higher anthocyanin intake is associated with lower arterial stiffness and central blood pressure in women. Am. J. Clin. Nutr. 96: 781-788.

Jennings, A., Welch, A.A., Spector, T., Macgregor, A., and Cassidy, A. (2014). Intakes of anthocyanins and flavones are associated with biomarkers of insulin resistance and inflammation in women. J. Nutr. 144 202-208.

Johnson, M.H., de Mejia, E.G., Fan, J., Lila, M.A., and Yousef, G.G. (2013) Anthocyanins and proanthocyanidins from blueberry-blackberry fermented beverages inhibit markers of inflammation in macrophage and carbohydrate-utilizing enzymes in vitro. Mol. Nutr. Food Res. 57: 1182-1197.

Kaume, L., Gbur, E.E., DiBrezzo, R., Howard, L.R., and Devareddy, L. (2014). Antioxidant-rich berries exert modest bone protective effects in postmenopausal smokers without improving biomarkers of bone metabolism. J. Funct. Foods 9: 202-210.

Kaume, L., Gilbert, W.C., Brownmiller, C., Howard, L.R., and Devareddy,

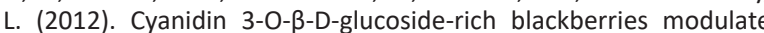
hepatic gene expression, and anti-obesity effects in ovariectomized rats. J. Funct. Foods 4: 480-488.

Kay, C.D., Kroon, P.A., and Cassidy, A. (2009). The bioactivity of dietary anthocyanins is likely to be mediated by their degradation products. Mol. Nutr. Food Res. 53: S92-S101.

Kellett, M.E., Greenspan, P., and Pegg, R.B. (2018). Modification of the cellular antioxidant activity (CAA) assay to study phenolic antioxidants in a Caco-2 cell line. Food Chem. 244: 359-363.

Kolniak-Ostek, J., Kucharska, A.Z., Sokoł-Łętowska, A., and Fecka, I. (2015). Characterization of phenolic compounds of thorny and thornless blackberries. J. Agric. Food Chem. 63: 3012-3021.

Komorsky-Lovrić, Š., and Novak, I. (2011). Determination of ellagic acid in 
strawberries, raspberries and blackberries by square-wave voltammetry. Int. J. Electrochem. Sci. 6: 4638-4647.

Larrosa, M., García-Conesa, M.T., Espín, J.C., and Tomás-Barberán, F.A. (2010). Ellagitannins, ellagic acid and vascular health. Mol. Aspects Med. 31: 513-539.

Liao, X., Greenspan, P., Srivastava, A., and Pegg, R.B. (2020). Characterizing the phenolic constituents of U.S. Southeastern blackberry cultivars. J. Berry Res. doi:10.3233/JBR-190475preprint.

Lipińska, L., Klewicka, E., and Sójka, M. (2014). Structure, occurrence and biological activity of ellagitannins: A general review. Acta Sci. Pol., Technol. Aliment. 13: 289-299.

Ma, G., and Chen, Y. (2020). Polyphenol supplementation benefits human health via gut microbiota: A systematic review via meta-analysis. J. Funct. Foods 66: 103829

Ma, H., Johnson, S.L., Liu, W., DaSilva, N.A., Meschwitz, S., Dain, J.A., and Seeram, N.P. (2018). Evaluation of polyphenol anthocyanin-enriched extracts of blackberry, black raspberry, blueberry, cranberry, red raspberry, and strawberry for free radical scavenging, reactive carbonyl species trapping, anti-glycation, anti- $\beta$-amyloid aggregation, and microglial neuroprotective effects. Int. J. Mol. Sci. 19: 461.

Manach, C., Williamson, G., Morand, C., Scalbert, A., and Rémésy, C. (2005). Bioavailability and bioefficacy of polyphenols in humans. I. Review of 97 bioavailability studies. Am. J. Clin. Nutr. 81: 230S-242S.

Mastroiacovo, D., Kwik-Uribe, C., Grassi, D., Necozione, S., Raffaele, A. Pistacchio, L., Righetti, R., Bocale, R., Lechiara, M.C., Marini, C., Ferri, C., and Desideri, G. (2015). Cocoa flavanol consumption improves cognitive function, blood pressure control, and metabolic profile in elderly subjects: the Cocoa, Cognition, and Aging (CoCoA) Study - a randomized controlled trial. Am. J. Clin. Nutr. 101: 538-548.

Mertz, C., Cheynier, V., Günata, Z., and Brat, P. (2007). Analysis of phenolic compounds in two blackberry species (Rubus glaucus and Rubus adenotrichus) by high-performance liquid chromatography with diode array detection and electrospray ion trap mass spectrometry. J. Agric. Food Chem. 55: 8616-8624

Mikulic-Petkovsek, M., Koron, D., Zorenc, Z., and Veberic, R. (2017). Do optimally ripe blackberries contain the highest levels of metabolites? Food Chem. 215: 41-49.

Milenkovic, D., Vanden Berghe, W., Boby, C., Leroux, C., Declerck, K., Szarc vel Szic, K., Heyninck, K., Laukens, K., Bizet, M., Defrance, M., Dedeurwaerder, S., Calonne, E., Fuks, F., Haegeman, G., Haenen, G.R.M.M., Bast, A., and Weseler, A.R. (2014). Dietary flavanols modulate the transcription of genes associated with cardiovascular pathology without changes in their DNA methylation state. PLoS ONE 9: e95527.

National Center for Complementary and Integrative Health. (2013). Antioxidants and Health: An Introduction. Retrieved March 30, 2020 from https://nccih.nih.gov/health/antioxidants/introduction.htm\# science.

Niculescu, V., Paun, N., Silion, M., and Popa, M.I. (2013). Anthocyanin profile of blackberries and grapes. Progress of Cryogenics and Isotopes Separation 16: 119-124.

Nile, S.H., and Park, S.W. (2014). Edible berries: Bioactive components and their effect on human health. Nutr. 30: 134-144.

Olas, B. (2018). Berry phenolic antioxidants - Implications for human health? Front. Pharmacol. 9: 78.

Ou, K., and Gu, L. (2014). Absorption and metabolism of proanthocyanidins. J. Funct. Foods 7: 43-53.

Pantelidis, G.E., Vasilakakis, M., Manganaris, G.A., and Diamantidis, G.r. (2007). Antioxidant capacity, phenol, anthocyanin and ascorbic acid contents in raspberries, blackberries, red currants, gooseberries and Cornelian cherries. Food Chem. 102: 777-783.

Park, S.H., Hanning, I., Gilbert, W., Munro, M., Devareddy, L., and Ricke, S.C. (2013). Feeding mice aged and fresh blackberries powder supplements result in shifts in the gastrointestinal microflora. Food $\mathrm{Bi}$ osci. 1: 66-72.

Rios, L.Y., Bennett, R.N., Lazarus, S.A., Rémésy, C., Scalbert, A., and Williamson, G. (2002). Cocoa procyanidins are stable during gastric transit in humans. Am. J. Clin. Nutr. 76: 1106-1110.

Sangiovanni, E., Vrhovsek, U., Rossoni, G., Colombo, E., Brunelli, C., Brembati, L., Trivulzio, S., Gasperotti, M., Mattivi, F., Bosisio, E., and Dell'Agli, M. (2013). Ellagitannins from Rubus berries for the control of gastric inflammation: In vitro and in vivo studies. PLOS ONE 8: e71762.

Schulz, M., Seraglio, S.K.T., Della Betta, F., Nehring, P., Valese, A.C., Daguer, H., Gonzaga, L.V., Oliveira Costa, A.C., and Fett, R. (2019). Blackberry (Rubus ulmifolius Schott): Chemical composition, phenolic compounds and antioxidant capacity in two edible stages. Food Res. Int. 122: 627-634.

Sellappan, S., Akoh, C.C., and Krewer, G. (2002). Phenolic compounds and antioxidant capacity of Georgia-grown blueberries and blackberries. J. Agric. Food Chem. 50: 2432-2438.

Serra, D., Paixão, J., Nunes, C., Dinis, T.C.P., and Almeida, L.M. (2013). Cyanidin-3-glucoside suppresses cytokine-induced inflammatory response in human intestinal cells: Comparison with 5-aminosalicylic acid. PLoS ONE 8: e73001.

Siriwoharn, T., and Wrolstad, R.E. (2004). Polyphenolic composition of Marion and Evergreen blackberries. J. Food Sci. 69: FCT233-FCT240.

Siriwoharn, T., Wrolstad, R.E., Finn, C.E., and Pereira, C.B. (2004). Influence of cultivar, maturity, and sampling on blackberry (Rubus L. hybrids) anthocyanins, polyphenolics, and antioxidant properties. J. Agric. Food Chem. 52: 8021-8030.

Solverson, P.M., Rumpler, W.V., Leger, J.L., Redan, B.W., Ferruzzi, M.G., Baer, D.J., Castonguay, T.W., and Novotny, J.A. (2018). Blackberry feeding increases fat oxidation and improves insulin sensitivity in overweight and obese males. Nutrients 10: 1048.

Spínola, V., Pinto, J., Llorent-Martínez, E.J., Tomás, H., and Castilho, P.C. (2019). Evaluation of Rubus grandifolius L. (wild blackberries) activities targeting management of type- 2 diabetes and obesity using in vitro models. Food Chem. Toxicol. 123: 443-452.

Ştefănuţ, M.N., Căta, A., Pop, R., Moşoarcă, C., and Zamfir, A.D. (2011). Anthocyanins HPLC-DAD and MS characterization, total phenolics, and antioxidant activity of some berries extracts. Anal. Lett. 44 2843-2855.

Ştefănuţ, M.N., Căta, A., Pop, R., Tănasie, C., Boc, D., lenaşcu, I., and Ordodi, V. (2013). Anti-hyperglycemic effect of bilberry, blackberry and mulberry ultrasonic extracts on diabetic rats. Plant Foods Hum. Nutr. 68: 378-384.

Tavares, L., Figueira, I., Macedo, D., McDougall, G.J., Leitão, M.C., Vieira, H.L.A., Stewart, D., Alves, P.M., Ferreira, R.B., and Santos, C.N. (2012) Neuroprotective effect of blackberry (Rubus sp.) polyphenols is potentiated after simulated gastrointestinal digestion. Food Chem. 131: 1443-1452.

Tavares, L., Figueira, I., Mcdougall, G.J., Vieira, H.L.A., Stewart, D., Alves, P.M., Ferreira, R.B., and Santos, C.N. (2013). Neuroprotective effects of digested polyphenols from wild blackberry species. Eur. J. Nutr. 52: 225-236.

Tomas, M., Rocchetti, G., Ghisoni, S., Giuberti, G., Capanoglu, E., and Lucini, L. (2020). Effect of different soluble dietary fibres on the phenolic profile of blackberry puree subjected to in vitro gastrointestinal digestion and large intestine fermentation. Food Res. Int. 130: 108954

Tomás-Barberán, F.A., García-Villalba, R., González-Sarrías, A., Selma, M.V., and Espín, J.C. (2014). Ellagic acid metabolism by human gut microbiota: Consistent observation of three urolithin phenotypes in intervention trials, independent of food source, age, and health status. J. Agric. Food Chem. 62: 6535-6538.

Van de Velde, F., Grace, M.H., Esposito, D., Pirovani, M.É., and Lila, M.A. (2016). Quantitative comparison of phytochemical profile, antioxidant, and anti-inflammatory properties of blackberry fruits adapted to Argentina. J. Food Compos. Anal. 47: 82-91.

Vasco, C., Riihinen, K., Ruales, J., and Kamal-Eldin, A. (2009). Phenolic compounds in Rosaceae fruits from Ecuador. J. Agric. Food Chem. 57: 1204-1212.

Wedick, N.M., Pan, A., Cassidy, A., Rimm, E.B., Sampson, L., Rosner, B. Willett, W., Hu, F.B., Sun, Q., and van Dam, R.M. (2012). Dietary flavonoid intakes and risk of type 2 diabetes in US men and women. Am. J. Clin. Nutr. 95: 925-933.

Welch, A., MacGregor, A., Jennings, A., Fairweather-Tait, S., Spector, T., and Cassidy, A. (2012). Habitual flavonoid intakes are positively associated with bone mineral density in women. J. Bone Miner. Res. 27: $1872-1878$

Wiese, S., Gärtner, S., Rawel, H.M., Winterhalter, P., and Kulling, S.E. (2009). Protein interactions with cyanidin-3-glucoside and its influence on $\alpha$-amylase activity. J. Sci. Food Agric. 89: 33-40. 
Yang, M., Koo, S.I., Song, W.O., and Chun, O.K. (2011). Food matrix affecting anthocyanin bioavailability: Review. Curr. Med. Chem. 18: 291-300.

Zhang, L., Zhou, J., Liu, H., Khan, M.A., Huang, K., and Gu, Z. (2012). Compositions of anthocyanins in blackberry juice and their thermal deg- radation in relation to antioxidant activity. Eur. Food Res. Technol. 235: 637-645.

Zunino, S.J., Zhang, Y., Seeram, N.P., and Storms, D.H. (2010). Berry fruit extracts inhibit growth and induce apoptosis of high-risk acute lymphoblastic leukemia cells in vitro. J. Funct. Foods 2: 187-195. 